\title{
Interaction between peroxisome proliferator-activated receptor gamma polymorphism and obesity on type 2 diabetes in a Chinese Han population
}

\author{
Xiaohui Lv ${ }^{1 \dagger}$, Li Zhang $^{1 \dagger}$, Jiayu Sun ${ }^{1}$, Zhigang Cai', Qing Gu', Ruipeng Zhang ${ }^{2}$ and Aiyun Shan ${ }^{1 *}$
}

\begin{abstract}
Aims: The aim of this study was to investigate the association of four single nucleotide polymorphisms (SNPs) of peroxisome proliferator-activated receptor gamma (PPARG) with type 2 diabetes mellitus (T2DM) risk and additional role of gene-obesity interaction.

Methods: Four SNPs were selected for genotyping in the case-control study: rs1805192, rs709158, rs3856806 and rs4684847. Generalized multifactor dimensionality reduction (GMDR) model and logistic regression was used to examine the interaction between SNP and obesity on T2DM, odds ratio (OR) and 95\% confident interval (95\% Cl) were calculated.

Results: T2DM risk was significantly higher in individuals with rs1805192-G allele $(p<0.05)$. The carriers of $\mathrm{G}$ allele of the rs 1805192 polymorphism revealed increased T2DM risk than those with CC variants (CG + GG versus CC, adjusted OR (95\% Cl) $1.76(1.45-2.06), p<0.001)$. T2DM risk was also significantly higher in individuals with rs3856806-T allele $(p<0.05)$. The carriers of T allele of the rs 3856806 polymorphism revealed increased T2DM risk than those with CC variants (CT + TT versus CC, adjusted OR (95\% Cl) 1.25 (1.17-1.76), $p<0.001)$. There was a significant two-locus model ( $p=0.0107$ ) involving rs 1805192 and obesity. Obese subjects with CG or GG genotype have the highest T2DM risk, compared to subjects with CC genotype and normal BMI (OR 2.40, 95\% Cl 1.68-3.63).

Conclusions: Our results support an important association between rs1805192 and rs3856806 minor allele (G allele) of PPARG and increased T2DM risk, the interaction analysis shown a combined effect of G- obesity interaction between rs1805192 and obesity on increased T2DM risk.
\end{abstract}

Keywords: Type 2 diabetes mellitus, PPAR, Polymorphism, Obesity, Interaction

\section{Background}

Diabetes mellitus (DM) is a group of common metabolic disorders that share the phenotype of hyperglycemia. Globally, about $5.4 \%$ of adult population has been estimated to have type 2 diabetes mellitus (T2DM) [1]. Several types of diabetes mellitus exist and are caused by a

\footnotetext{
*Correspondence: zhaocgcg2816@163.com

†Xiaohui Lv and Li Zhang contributed equally to this work

1 Shenzhen Futian District Traditional Chinese Medicine Hospital, No. 6001

in North Central Avenue, Futian District, Shenzhen 518033, Guangdong,

China

Full list of author information is available at the end of the article
}

complex interaction of genetics and environmental factors [2]. Type 2 diabetes has a strong genetic component. There is evidence that the Pro12Ala polymorphism is linked to obesity and T2DM [3].

The peroxisome proliferator-activated receptor gamma (PPARG) is now recognized to play a main PPARG entered the spotlight as a major player in metabolic regulation in the early 1990s [4] through the discovery of thiazolidinediones (TZDs) as potent synthetic insulin-sensitizing drugs. TZDs quickly passed clinical trials and became front-line agents in the treatment of type II diabetes for their robust insulin-sensitizing and 
glucose-lowering actions, although their popularity has recently decreased in response to safety concerns. Activation of PPARG results in systemic insulin sensitization through complex mechanisms involving multiple. PPARG was the first gene reproducibly associated with T2DM. The association between the substitution of alanine by proline at codon 12 of PPARG2 (Ala12 allele) and the risk for T2DM has been widely studied since Yen et al. [5], first reported this polymorphism. Some studies indicated that rs1805192 SNP plays a key role in regulating the expression of numerous genes involved in lipid metabolism, metabolic syndrome, inflammation, and atherosclerosis [6, 7]. Some environmental risk factors for T2DM were suggested, such as obesity, which was reported in different populations [8-10]. However, till now, no study focused on the impact of gene- environment interaction between PPARG and obesity on T2DM risk in Chinese population. So the aim of this study was to investigate the association of PPARG, and additional PPARG gene- obesity interaction with T2DM risk based on a Chinese population.

\section{Methods Subjects}

This was a case-control study. Chinese patients with T2DM were consecutively recruited between January 2012 and December 2013 from the Shenzhen Futian District traditional Chinese medicine hospital. A total of 1297 subjects consist of 647 T2DM patients and 650 normal controls were included in this study, including 606 males and 691 females. The mean age of all subjects was $54.3 \pm 15.8$ years old. The selected subjects were similar to those who were not selected in terms of age, sex, smoking status and alcohol consumption. Informed consent was obtained from all participants.

\section{Body measurements}

Data on general demographic information and lifestyle information for all participants were obtained using a standard questionnaire administered by trained staffs. Body weight, height and waist circumference (WC) was measured, and body mass index (BMI) was calculated as weight in kilograms divided by the square of the height in meters. Cigarette smokers were those who self-reported smoking cigarettes at least once a day for 1 year or more [11]. Alcohol consumption was expressed as the sum of milliliters of alcohol per week from wine, beer, and spirits [12]. Blood samples were collected in the morning after at least $8 \mathrm{~h}$ of fasting. All plasma and serum samples were frozen at $-80{ }^{\circ} \mathrm{C}$ until laboratory testing. Plasma glucose was measured using an oxidase enzymatic method. Concentrations of high-density lipoprotein (HDL)-cholesterol and triglyceride (TG) were assessed enzymatically by an automatic biochemistry analyzer (Hitachi Inc, Tokyo, Japan) using commercial reagents. All analysis was performed by the same lab.

\section{Genomic DNA extraction and genotyping}

We selected SNPs within PPARG according to the following methods: (1) more studied SNPs, such as rs1805192, which was more studied in previous studies; (2) we also selected the others SNP of PPARG, in order to find new SNP associated with T2DM in Chinese population. Four SNPs were selected for genotyping in this study: rs1805192, rs709158, rs3856806, rs4684847. Four SNPs were detected by Taqman fluorescence probe, and probe sequences of four SNPs were shown in Table 1. Genomic DNA from participants was extracted from EDTAtreated whole blood, using the DNA Blood Mini Kit (Qiagen, Hilden, Germany) according to the manufacturer's instructions. ABI Prism7000 software and allelic discrimination procedure was used for genotyping of forementioned four SNPs. A $25 \mu \mathrm{l}$ reaction mixture including $1.25 \mu \mathrm{l}$ SNP Genotyping Assays $(20 \times), 12.5 \mu \mathrm{l}$ Genotyping Master Mix $(2 \times), 20$ ng DNA, and the conditions were as follows: initial denaturation for $10 \mathrm{~min}$ and $95^{\circ} \mathrm{C}$, denaturation for $15 \mathrm{~s}$ and $92{ }^{\circ} \mathrm{C}$, annealing and extension for $90 \mathrm{~s}$ and $60^{\circ} \mathrm{C}, 50$ cycles.

\section{Diagnostic criteria}

Diagnosis of diabetes at baseline for a fasting glucose was $\geq 126 \mathrm{mg} / \mathrm{dl}$ ( $7.0 \mathrm{mmol} / \mathrm{l})$ or if hypoglycemic therapy (oral agents or insulin) had been executed. During the follow-up, the criteria for the diagnosis of T2DM included a fasting glucose $\geq 126 \mathrm{mg} / \mathrm{dl}(7.0 \mathrm{mmol} / \mathrm{l})$, or a $2 \mathrm{~h}$ postprandial blood glucose $\geq 200 \mathrm{mg} / \mathrm{dl}(11.0 \mathrm{mmol} / \mathrm{l})$, or if hypoglycemic therapy (oral agents or insulin) had been started in the interim [13].

Obesity was defined by using WHO criteria for Asian populations: $B M I$ value $\geq 28 \mathrm{~kg} / \mathrm{m}^{2}$ [14].

\section{Statistical analysis}

The means and SD (standard deviation) was calculated for normally distributed continuous variables, and compared using t test. Percentages were calculated for categorical variable, and compared between case and control group participants using a Chi square test. Genotype and allele frequencies were obtained by direct count. Genotype distributions in T2DM patients and controls were evaluated by $\chi^{2}$ test using SPSS (version 19.0; SPSS Inc., Chicago, IL). Hardy-Weinberg equilibrium (HWE) was performed by using SNPStats (available online at http:// bioinfo.iconcologia.net/SNPstats). Logistic regression model was used to examine the interaction between SNP and obesity on T2DM in additive model, odds ratio (OR) and $95 \%$ confident interval $(95 \% \mathrm{CI})$ were calculated. 
Table 1 Probe sequence for four SNPs used for Taqman fluorescence probe analysis

\begin{tabular}{|c|c|c|c|c|c|}
\hline SNP & Rs number & Chromosome & Functional consequence & Position & Probe sequence \\
\hline C1341T & rs3856806 & 3 & Intron variant & $12,415,557$ & $\begin{array}{l}\text { 5'-GGTTGACACAGAGATGCCATTCTGG[C/G] } \\
\text { CCACCAACTTTGGGATCAGCTCCGT-3' }\end{array}$ \\
\hline Intron $A>G$ & rs709158 & 3 & Intron variant & $12,403,176$ & $\begin{array}{l}\text { 5'-AGATACGGGGGAGGAAATTCACTGG[A/G] } \\
\text { TTTTACAATATATTTTTCAAGGCAA-3' }\end{array}$ \\
\hline Pro12Ala & rs1805192 & 3 & Missense & $12,361,238$ & $\begin{array}{l}\text { 5'-ACCTCAGACAGATTGTCACGGAACA[C/T] } \\
\text { GTGCAGCTACTGCAGGTGATCAAGA-3' }\end{array}$ \\
\hline Intron $C>T$ & rs4684847 & 3 & Intron variant & $12,326,337$ & $\begin{array}{l}\text { 5'-ATTTATTTAAATCATCTCTAATTCT[C/T] } \\
\text { ACAACTCCGAAAAGATAAGAAAACA-3' }\end{array}$ \\
\hline
\end{tabular}

Odds were adjusted for gender, age, smoke and alcohol status, high fat diet, low fiber diet, TC, TG and HDL. Generalized multifactor dimensionality reduction (GMDR) [15] was used to investigate the gene- environment interaction, cross-validation consistency, the testing balanced accuracy, and the sign test, to assess each selected interaction were calculated. The cross-validation consistency score is a measure of the degree of consistency with which the selected interaction is identified as the best model among all possibilities considered. Testing-balanced accuracy is a measure of the degree to which the interaction accurately predicts case-control status, and yields a score between 0.50 (indicating that the model predicts no better than chance) and 1.00 (indicating perfect prediction). Finally, the sign test, or permutation test (providing empirical $\mathrm{p}$ values), for prediction accuracy can be used to measure the significance of an identified model.

\section{Results}

A total of 1297 subjects including 647 T2DM patients and 650 normal controls were included in this study, including 606 males and 691 females. The mean age of all subjects was $54.3 \pm 15.8$ years old. Participants characteristics stratified by T2DM cases and controls are shown in Table 2. The distributions of males, smoking, alcohol consumption, high fat diet, low fiber diet and mean of age were not different between cases and controls. The means of BMI, WC, TG, TC and HDL were significantly different between cases and controls.

All genotypes were distributed according to HardyWeinberg equilibrium (all p values were more than 0.05). There were significant differences in rs1805192 and rs3856806 alleles and genotypes distributions between cases and controls (Table 3). The frequency for $\mathrm{G}$ allele of rs1805192 was higher in cases $(49.0 \%$ in T2DM patients and $37.5 \%$ in controls, $\mathrm{p}<0.001)$. Logistic analysis showed that T2DM risk was significantly higher in individuals with rs1805192-G allele ( $\mathrm{p}<0.05)$. The carriers of $G$ allele of the rs1805192 polymorphism revealed increased T2DM risk than those with $\mathrm{CC}$ variants (CG + GG versus CC, adjusted OR (95\% CI) 1.76 (1.45$2.06), \mathrm{p}<0.001)$. The frequency for $\mathrm{T}$ allele of $\mathrm{rs} 3856806$ was higher in cases $(50.2 \%$ in T2DM patients and $38.9 \%$ in controls, $\mathrm{p}<0.001)$. Logistic analysis showed that T2DM risk was significantly higher in individuals with rs3856806-T allele $(p<0.05)$. The carriers of $\mathrm{T}$ allele of the rs3856806 polymorphism revealed increased T2DM risk than those with $\mathrm{CC}$ variants $(\mathrm{CT}+\mathrm{TT}$ versus $\mathrm{CC}$, adjusted OR (95\% CI) 1.25 (1.17-1.76), p < 0.001). However, we did not find any significant association between other two SNPs and T2DM risk before or after covariates adjustment.

We employed the GMDR analysis to assess the impact of the PPARG gene- obesity interaction on T2DM risk, after adjustment for gender, age, smoke and alcohol consumption status, high fat diet, low fiber diet, TC and HDL. Table 4 summarizes the results obtained from GMDR analysis for two- to five-locus models. There was a significant two-locus model ( $p=0.0107)$ involving rs1805192 and obesity, indicating a potential geneenvironment interaction among rs1805192 and obesity. Overall, the two- locus models had a cross-validation consistency of 10 of 10 , and had the testing accuracy of $62.17 \%$.

In order to obtain the odds ratios and 95\% CI for the joint effects of rs1805192 genotype and obesity on T2DM, we conducted interaction analysis between rs1805192 and obesity. We found that obese subjects with CG or GG genotype have the highest T2DM risk, compared to subjects with CC genotype and normal BMI (OR 2.40, 95\% CI 1.68-3.63), after adjustment for gender, age, smoke and alcohol status, high fat diet, low fiber diet, TC and HDL (Table 5).

\section{Discussion}

The result of this study indicated that rs1805192- G allele and rs3856806-T allele of PPARG is significantly associated with higher T2DM risk, however, we did not find any association between the others SNPs and 
Table 2 General characteristics of study participants in T2DM case and control group

\begin{tabular}{llll}
\hline Variables & T2DM cases group $(\mathbf{n = 6 4 7 )}$ & Control group $(\mathbf{n}=\mathbf{6 5 0})$ & $\boldsymbol{p}$ values \\
\hline Age (years) & $56.7 \pm 14.8$ & $55.4 \pm 14.2$ & 0.107 \\
Males N (\%) & $310(47.9)$ & $296(45.5)$ & 0.391 \\
Smoke N (\%) & $153(23.6)$ & $174(26.8)$ & 0.257 \\
Alcohol consumption N (\%) & $144(22.2)$ & $136(20.9)$ & 0.559 \\
High fat diet N (\%) & $106(16.4)$ & $114(17.5)$ & 0.579 \\
Low fiber diet N (\%) & $118(18.2)$ & $96(14.8)$ & 0.092 \\
WC $(\mathrm{cm})$ & $89.1 \pm 15.8$ & $86.8 \pm 16.5$ & 0.010 \\
BMI (kg/m $\left.{ }^{2}\right)$ & $25.8 \pm 6.8$ & $23.3 \pm 6.6$ & $<0.001$ \\
TG $(\mathrm{mmol} / \mathrm{l})$ & $2.1 \pm 0.67$ & $1.8 \pm 0.70$ & $<0.001$ \\
TC $(\mathrm{mmol} / \mathrm{l})$ & $5.3 \pm 1.2$ & $4.7 \pm 1.1$ & $<0.001$ \\
HDL $(\mathrm{mmol} / \mathrm{l})$ & $1.21 \pm 0.33$ & $1.32 \pm 0.27$ & $<0.001$ \\
\hline
\end{tabular}

Median and inter quartile for TG; mean \pm standard deviation for age, FPG, TC, HDL-C

$T C$ total cholesterol, HDL high density lipoprotein, FPG fast plasma glucose, TG triglyceride

Table 3 Genotype and allele frequencies of four SNPs between T2DM case and control group

\begin{tabular}{|c|c|c|c|c|c|c|}
\hline \multirow[t]{2}{*}{ SNPs } & \multirow[t]{2}{*}{ Genotypes and Alleles } & \multicolumn{2}{|c|}{ Frequencies $\mathrm{N}(\%)$} & \multirow[t]{2}{*}{ OR $(95 \% \mathrm{Cl})^{\mathrm{a}}$} & \multirow[t]{2}{*}{$p$ values } & \multirow[t]{2}{*}{ HWE test } \\
\hline & & Case $(n=647)$ & Control $(n=650)$ & & & \\
\hline \multirow[t]{6}{*}{ rs3856806 } & $\mathrm{CC}$ & $322(49.8)$ & $397(61.1)$ & 1.00 & $<0.001$ & 0.379 \\
\hline & $\mathrm{CT}$ & $256(39.6)$ & $217(33.4)$ & $1.12(1.06-1.58)$ & & \\
\hline & $\mathrm{TT}$ & $69(10.7)$ & $36(5.5)$ & $1.93(1.34-2.71)$ & & \\
\hline & $\mathrm{CT}+\mathrm{TT}$ & $325(50.2)$ & $253(38.9)$ & $1.25(1.17-1.76)$ & $<0.001$ & \\
\hline & C & $900(69.6)$ & $1011(77.8)$ & & $<0.001$ & \\
\hline & $\mathrm{T}$ & $394(30.4)$ & $289(22.2)$ & & & \\
\hline \multirow[t]{6}{*}{ rs709158 } & $\mathrm{AA}$ & $341(52.7)$ & $373(57.4)$ & 1.00 & 0.128 & 0.165 \\
\hline & $A G$ & $243(37.6)$ & $230(35.4)$ & $1.06(0.95-1.37)$ & & \\
\hline & GG & $63(9.7)$ & $47(7.2)$ & $1.10(0.86-1.61)$ & & \\
\hline & $A G+G G$ & $306(47.3)$ & $277(42.6)$ & $1.07(0.92-1.43)$ & 0.090 & \\
\hline & A & $925(71.5)$ & $976(75.1)$ & & 0.039 & \\
\hline & G & $369(28.5)$ & $324(24.9)$ & & & \\
\hline \multirow[t]{6}{*}{ rs1805192 } & $\mathrm{CC}$ & $330(51.0)$ & $406(62.5)$ & 1.00 & $<0.001$ & 0.385 \\
\hline & $C G$ & $262(40.5)$ & $220(33.8)$ & $1.42(1.61-1.84)$ & & \\
\hline & GG & $55(8.5)$ & $24(3.7)$ & $2.13(1.72-2.93)$ & & \\
\hline & $C G+G G$ & $317(49.0)$ & $244(37.5)$ & $1.76(1.45-2.06)$ & $<0.001$ & \\
\hline & $\mathrm{C}$ & $922(71.2)$ & $1032(79.4)$ & & $<0.001$ & \\
\hline & G & $372(28.8)$ & 268 (20.6) & & & \\
\hline \multirow[t]{6}{*}{ rs4684847 } & $\mathrm{CC}$ & $346(53.5)$ & $379(58.3)$ & 1.00 & 0.203 & 0.277 \\
\hline & $\mathrm{CT}$ & $250(38.6)$ & $228(35.1)$ & $1.08(0.91-1.36)$ & & \\
\hline & $\mathrm{TT}$ & $51(7.9)$ & $43(6.6)$ & $1.04(0.82-1.53)$ & & \\
\hline & $\mathrm{CT}+\mathrm{TT}$ & $301(46.5)$ & $271(41.7)$ & $1.07(0.89-1.39)$ & 0.080 & \\
\hline & C & $942(72.8)$ & $986(75.8)$ & & 0.076 & \\
\hline & $\mathrm{T}$ & $352(27.2)$ & $314(24.2)$ & & & \\
\hline
\end{tabular}

${ }^{a}$ Adjusted for gender, age, smoke and alcohol consumption status, high fat diet, low fiber diet, TC and HDL

T2DM. Although many studies have taken a candidate gene approach to investigate the genetic etiology of T2DM, implicating the potential candidate genes in T2DM risk factors, which include genes for rs1805192 and rs3856806 polymorphism of PPARG [16], however the results of association between rs1805192 and T2DM are inconsistent. Wang et al. [17] demonstrated that the presence of the Ala allele may contribute to improved 
Table 4 Best gene- obesity interaction models, as identified by GMDR

\begin{tabular}{lllll}
\hline Locus no. & Best combination & Cross-validation consistency & Testing accuracy & p values* \\
\hline 2 & rs1805192 Obesity & $10 / 10$ & 0.6217 & 0.0107 \\
3 & rs1805192 rs3856806 Obesity & $9 / 10$ & 0.5577 & 0.1719 \\
4 & rs1805192 rs3856806 rs709158 Obesity & $8 / 10$ & 0.5590 & 0.0547 \\
5 & rs1805192 rs3856806 rs709158 rs4684847 Obesity & $7 / 10$ & 0.4958 & 0.3770 \\
\hline
\end{tabular}

* Adjusted for gender, age, smoke and alcohol consumption status, high fat diet, low fiber diet, TC and HDL

Table 5 Interaction analysis for rs1805192 and obesity on T2DM by using logistic regression

\begin{tabular}{llll}
\hline Rs1805192 & Obesity & OR $(\mathbf{9 5 \%} \mathbf{C l})^{\mathbf{a}}$ & $\boldsymbol{p}$ values \\
\hline CC & No & 1.00 & - \\
CG or GG & No & $1.21(1.08-1.47)$ & 0.020 \\
CC & Yes & $1.52(1.13-1.93)$ & $<0.001$ \\
CG or GG & Yes & $2.40(1.68-3.63)$ & $<0.001$
\end{tabular}

a Adjusted for gender, age, smoke and alcohol consumption status, high fat diet, low fiber diet, TC and HDL

insulin secretory capacity and may confer protection from T2DM and obesity in the Chinese population. Moreover, a meta-analysis confirmed the association between the PPARG2 Pro12 allele and T2DM, and suggested that patients who carry the Pro12 allele have a 1.27-fold higher risk for developing T2DM than Ala12 carriers. This seemingly modest effect translates into a staggering $25 \%$ population-attributable risk because of the higher frequency of the Pro12 allele, especially in Japanese and European populations [18]. However, Bener et al. [19] suggested that no significant association between the Pro12Ala polymorphism of the PPARG2 gene and T2DM in Qatari's population. Al-Safar et al. [20] also suggested that confirmed that Pro12Ala mutation in PPARG2 is not associated with T2DM risk in this population. In a study for South Africa population, Vergotine et al. [21] reported that the Pro12Ala of PPARG2 is significantly associated with insulin resistance and this polymorphism interacts with IRS1 Gly972Arg, to increase the risk of T2DM. Hahn et al. [22] indicated that our data confirm a beneficial effect of the PPARG1 12Ala allele on insulin resistance and glucose metabolism in PCOS women. Furthermore, the Ala allele appears to be associated with less severe hirsutism, independent of hyperandrogenemia. Tellechea et al. [23] conducted a study for males of Argentinian blood donors of selfreported European ancestry and indicated that healthy men, in particular nonsmokers, carrying the Ala12 allele of PPARG rs1801282 polymorphism, have a high risk for metabolic syndrome (MetS) and insulin resistance (IR). Fan et al. [24] indicated that the genotypes with minor allele variants at the rs1805192, rs709158 and rs3856806 loci are associated with increased LDL-C levels, which was a risk factor of T2DM. Gu et al. [25] also suggested that in the codominant and log-additive models, rs1805192 and rs3856806 were all associated with increased dyslipidemia risk. A study conducted by Phani et al. [26] showed that PPARG2 variants are associated with increased T2DM susceptibility when associated with adiposity in Indian population. These data reflect an association of analyzed PPARG gene polymorphisms with values of insulin, HDL, LDL and total cholesterol which indicates an important role of these genes in lipid metabolism and pathogenesis of T2DM and MetS [16].

T2DM is associated with both environmental and genetic factors. Several metabolic abnormalities are implicated in its pathogenesis, such as obesity and other environmental factors. Several studies have confirmed the association between obesity and T2DM. However, till now, no study focused on impact of gene-environment interaction between PPARG and obesity on T2DM risk in Chinese population. This study investigated the impact of additional PPARG gene-obesity interaction on T2DM risk based on a Chinese population by using GMDR model. We found that a significant two-locus model involving rs1805192 and obesity, indicating a potential gene-environment interaction among rs1805192 and obesity. Obese subjects with CG or GG genotype have the highest T2DM risk, compared to subjects with CC genotype and normal BMI.

Several limitations of this study should be considered. Firstly, More SNPs of PPARG gene should been chosen. The limited SNPs were not sufficient to capture most genetic information of PPARG. Secondly, there was a relatively small sample size in the study, other larger sample studies should be conducted in the future. Thirdly, we did not obtain any information on IR level.

\section{Conclusions}

Our results support an important association between rs1805192 and rs3856806 minor allele (G allele) of PPARG and increased T2DM risk, the interaction analysis shown a combined effect of G- obesity interaction between rs1805192 and obesity on increased T2DM risk. 


\section{Authors' contributions}

$X \mathrm{~L}$ and $\mathrm{LZ}$ carried out the molecular genetic studies, participated in the sequence alignment and drafted the manuscript. JS carried out the immunoassays. ZC participated in the sequence alignment. QG participated in the design of the study and performed the statistical analysis. RZ and XL conceived of the study, and participated in its design and coordination and helped to draft the manuscript. All authors read and approved the final manuscript.

\section{Author details}

1 Shenzhen Futian District Traditional Chinese Medicine Hospital, No. 6001 in North Central Avenue, Futian District, Shenzhen 518033, Guangdong, China. ${ }^{2}$ Key Lab for New Drugs Research of TCM, Research Institute of Tsinghua University in Shenzhen, Shenzhen, Guangdong, China.

\section{Acknowledgements}

The writing of this paper was supported by Shenzhen Futian District traditional Chinese medicine hospital. We thank all the partners and staffs who help us in the process of this study. Lv-Xiaohui and ZHANG-Li contributed equally to this work.

\section{Competing interests}

The authors declare that they have no competing interests.

\section{Consent for publication}

We have obtained consent to publish from the participant.

\section{Ethics approval and consent to participate}

We have obtained consent to publish from the participant (or legal parent or guardian for children) to report individual patient data.

\section{Funding}

This paper was founded by Shenzhen Science and Technology R\&D Fund (No: JCYJ20140414145007216)

Received: 24 June 2016 Accepted: 13 January 2017

Published online: 19 January 2017

\section{References}

1. Mogre V, Salifu ZS, Abedandi R. Prevalence, components and associated demographic and lifestyle factors of the metabolic syndrome in type 2 diabetes mellitus. J Diabetes Metab Disord. 2014;13:80

2. Anthony FS, Braunwald E, Kasper DL. Harrison's principles of internal medicine. 17th ed. New York: McGraw Hill Publication; 2008. p. 2275.

3. Danawati CW, Nagata M, Moriyama H, Hara K, Yasuda H, Nakayama M, Kotani R, Yamada K, Sakata M, Kurohara M, Wiyono P, Asdie H, Sakaue M, Taniguchi H, Yokono K. A possible association of Pro12Ala polymorphism in peroxisome proliferator-activated receptor $\gamma 2$ gene with obesity in native Javanese in Indonesia. Diabetes Metab Res Rev. 2005;21:465-9.

4. Lehmann J, Moore L, Smith-Oliver T, Wilkison W, Willson T, Kliewer S. An antidiabetic thiazolidinedione is a high affinity ligand for peroxisome proliferator-activated receptor gamma (PPAR gamma). J Biol Chem. 1995:270:12953-6.

5. Yen CJ, Beamer BA, Negri C, Silver K, Brown KA, Yarnall DP, Burns DK, Roth J, Shuldiner AR. Molecular scanning of the human peroxisome proliferator activated receptor gamma (hPPAR gamma) gene in diabetic Caucasians: identification of a Pro12Ala PPAR gamma 2 missense mutation. Biochem Biophys Res Commun. 1997;241:270-4.

6. Argmann CA, Cock TA, Auwerx J. Peroxisome proliferator-activated receptor gamma: the more the merrier? Eur J Clin Invest. 2005;35:82-92.

7. Desvergne B. Be fit or be sick: peroxisome proliferator-activated receptors are down the road. Mol Endocrinol. 2004;18:1321-32.

8. National Institute of Health. Clinical guidelines on the identification, evaluation, and treatment of overweight and obesity in adults - the evidence report. Obes Res. 1998;6(Suppl 2):51S-209S

9. Asia Pacific Cohort Studies Collaboration, Ni Mhurchu C, Parag V, Nakamura M, Patel A, Rodgers A, Lam TH. Body mass index and risk of diabetes mellitus in the Asia-Pacific region. Asia Pac J Clin Nutr. 2006;15(2):127-33.
10. Schienkiewitz A, Schulze MB, Hoffmann K, Kroke A, Boeing H. Body mass index history and risk of type 2 diabetes: results from the European Prospective Investigation into Cancer and Nutrition (EPIC)-Potsdam Study. Am J Clin Nutr. 2006;84(2):427-33.

11. Iida H, Kumar JV, Kopycka-Kedzierawski DT, Billings RJ. Effect of tobacco smoke on the oral health of US. women of childbearing age. J Public Health Dent. 2009:69:231-41.

12. Freiberg MS, Cabral HJ, Heeren TC, Vasan RS, Ellison RC. Alcohol consumption and the prevalence of the metabolic syndrome in the US. Diabetes Care. 2004;27:2954-9.

13. Genuth S, Alberti KG, Bennett P, Buse J, Defronzo R, Kahn R, Kitzmiller J, Knowler WC, Lebovitz H, Lernmark A, Nathan D, Palmer J, Rizza R, Saudek C, Shaw J, Steffes M, Stern M, Tuomilehto J, Zimmet P. Expert Committee on the Diagnosis and Classification of Diabetes Mellitus. Follow-up report on the diagnosis of diabetes mellitus, The Expert Committee on the Diagnosis and Classification of Diabetes Mellitus. Diabetes Care. 2003;26:3160-7.

14. Consultation WHO. Obesity:Preventing and managing the global epidemic. Geneva: WHO Technical Report Series; 2000. p. 894.

15. Lou XY, Chen GB, Yan L, Ma JZ, Zhu J, Elston RC, Li MD. A generalized combinatorial approach for detecting gene-by gene and gene-by- environment interactions with application to nicotine dependence. Am J Hum Genet. 2007;80(6):1125-37.

16. Bego T, Dujic T, Mlinar B, Semiz S, Malenica M, Prnjavorac B, Ostanek B, Marc J, Causević A. Association of PPARG and LPIN1 gene polymorphisms with metabolic syndrome and type 2 diabetes. Med Glas (Zenica). 2011;8(1):76-83.

17. Wang X, Liu J, Ouyang Y, Fang M, Gao H, Liu L. The association between the Pro12Ala variant in the PPARY2 gene and type 2 diabetes mellitus and obesity in a Chinese population. PLOS ONE. 2013:8:e71985.

18. De Cosmo S, Prudente S, Lamacchia O, Lapice E, Morini E, Di Paola R, Copetti M, Ruggenenti P, Remuzzi G, Vaccaro O, Cignarelli M, Trischitta V. PPARY2 P12A polymorphism and albuminuria in patients with type 2 diabetes: a meta-analysis of case-control studies. Nephrol Dial Transplant. 2011;26:4011-6.

19. Bener A, Zirie M, Al-Hamaq A, Nawaz Z, Samson N, Mohammad R. Impact of the Pro12Ala polymorphism of the PPARY2 gene on diabetes and obesity in a highly consanguineous population. Indian J Endocrinol Metab. 2015;19(1):77-83.

20. Al-Safar H, Hassoun A, Almazrouei S, Kamal W, Afandi B, Rais N. Association of the genetic polymorphisms in transcription factor 7-like 2 and peroxisome proliferator-activated receptors- $\gamma 2$ with type 2 diabetes mellitus and its interaction with obesity status in Emirati Population. J Diabetes Res. 2015;2015:129695

21. Vergotine Z, Yako YY, Kengne AP, Erasmus RT, Matsha TE. Proliferatoractivated receptor gamma Pro12Ala interacts with the insulin receptor substrate 1 Gly972Arg and increase the risk of insulin resistance and diabetes in the mixed ancestry population from South Africa. BMC Genet. 2014;15:10.

22. Hahn S, Fingerhut A, Khomtsiv U, Khomtsiv L, Tan S, Quadbeck B, Herrmann BL, Knebel B, Müller-Wieland D, Mann K, Janssen OE. The peroxisome proliferator activated receptor gamma Pro12Ala polymorphism is associated with a lower hirsutism score and increased insulin sensitivity in women with polycystic ovary syndrome. Clin Endocrinol (Oxf). 2005;62(5):573-9

23. Tellechea ML, Aranguren F, Pérez MS, Cerrone GE, Frechtel GD, Taverna MJ. Pro12Ala polymorphism of the peroxisome proliferator- activated receptor- $\gamma$ gene is associated with metabolic syndrome and surrogate measures of insulin resistance in healthy men: interaction with smoking status. Circ J. 2009;73(11):2118-24

24. Fan W, Shen C, Wu M, Zhou ZY, Guo ZR. Association and interaction of PPARa, $\delta$, and $\gamma$ gene polymorphisms with low-density lipoproteincholesterol in a Chinese Han population. Genet Test Mol Biomark. 2015;19(7):379-86.

25. Gu SJ, Guo ZR, Zhou ZY, Hu XS, Wu M. PPAR a and PPAR y polymorphisms as risk factors for dyslipidemia in a Chinese Han population. Lipids Health Dis. 2014;13:23.

26. Phani NM, Vohra M, Rajesh S, Adhikari P, Nagri SK, D'Souza SC, Satyamoorthy K, Rai PS. Implications of critical PPARY2, ADIPOQ and FTO gene polymorphisms in type 2 diabetes and obesity- mediated susceptibility to type 2 diabetes in an Indian population. Mol Genet Genom. 2015. doi:10.1007/s00438-015-1097-4 